van der Pluijm, Gabri

[49]

\section{Monitoring metastatic behavior of human breast cancer cells in mice with species- specific PCR and bioluminescent reporter imaging}

G. van der Pluijm ${ }^{1}$, A. Wetterwald ${ }^{2}$, B. Sijmons ${ }^{1}$, E. Gautschi ${ }^{2}$ I. Que ${ }^{1,}$ B. Stadler ${ }^{3}$, G. Thalmann², M. Cecchini ${ }^{2} \&$ C. Löwik ${ }^{1}$

${ }^{1}$ Leiden University Medical Center, Dept. of Endocrinology, Leiden, The Netherlands

${ }^{2}$ Gene Therapy Laboratory, Department of Clinical Research and Urology Clinic, University Hospital, Bern, Switzerland

${ }^{3}$ Institute of Immunology and Allergology, University Hospital, Bern, Switzerland

Breast cancer metastasizes frequently to the skeleton and causes considerable morbidity. Injection of human MDA-MB-231 breast cancer cells into the left heart ventricle of immunodeficient mice produced multiple osteolytic bone lesions and soft tissue metastases. However, micrometastases are not readily detectable and more sensitive methods are required to detect minimal disease states. Here we describe the use of species-specific PCR (ssPCR) and bioluminescent reporter imaging (BRI) for monitoring the tumor metastatic in vivo. We used a CCD camera connected to the Argus-20 image processor (C-2400/VIM, Hamamatsu) to monitor bone metastasis by tumor cells transfected with the luciferase reporter gene. MDA-MB-231 cells were stably transfected with a PCMV-plasmid containing the firefly luciferase gene (MDA-231/luc+). After intracardiac inoclation with MDA-231/luct cells, the mice were monitored weekly for the development of bone and soft tissue metastases. For this, the mice were anaesthesized, injected with D-luciferin and photon emission was measured. Micrometastases developed predominantly in the hind limbs and the axial skeleton. Distinct photon emission was detected already after 24 days in bone (detection limit \pm 10.000 cells). ssPCR revealed significantly increased expression of VEGF-A, -B and PTHrP by the cancer cells in bone metastases when compared to soft tissues metastases suggesting a causal role in the observed preferential skeletal metastasis. In conclusion, BRI and ssPCR can be used for quantitative detection and growth of micrometastases and may become extremely useful to study the pathogenesis of bone metastasis in living animals, to monitor expression of targeted gene vectors and to determine the efficacy of novel therapeutic agents.

Vandesompele, Jo $[50]$

\section{Integration of suppression subtractive hybridization, laser capture microdissection and microarray analysis for identification of genes implicated in neuroblastoma pathogenesis}

Jo Vandesompele ${ }^{1}$, Nadine Van Roy ${ }^{1}$, Geneviève Laureys ${ }^{2}$, Katleen De Preter ${ }^{1}$, Geert Ber ${ }^{3}$, Kristin Strumane ${ }^{3}$, Anne De Paepe $^{1}$, Frans Van Roy ${ }^{3} \&$ Frank Speleman ${ }^{1}$

${ }^{1}$ Center for Medical Genetics, University Hospital, Ghent, Belgium

${ }^{2}$ Department of Pediatric Oncology, University Hospital, Ghent, Belgium

${ }^{3}$ Department of Molecular Biology, VIB, Belgium

Neuroblastoma is the most frequent extracranial solid tumor in children, showing remarkable clinical and genetic heterogeneity. To make further progress in understanding the genetic basis of neuroblastoma we have applied suppression subtractive hybridization for the identification of differentially expressed transcripts. We analyzed two neuroblastoma cell lines belonging to different genetic subgroups, respectively with and without $1 \mathrm{p}$ and $11 \mathrm{q}$ deletion and MYCN amplification. Northern blot analysis and real-time quantitative polymerase chain reaction with reverse transcription showed differential expression in $75 \%$ of the selected genes. We identified both rare and highly abundant differential transcripts. Known and previously unreported genes from the $2 \mathrm{p} 23$ and $2 \mathrm{p} 13-14$ amplified regions in IMR32 were efficiently selected. From the present set of suppression subtractive hybridization clones, we will select genes relevant to normal neurogenesis through expression analysis of normal fetal neuroblasts isolated by laser capture microdissection (Arcturus, PixCell II). We will select genes implicated in particular subsets of neuroblastoma by microarray analysis (Affymetrix 417) of a large panel of neuroblastoma cell lines and primary tumors.

Vandesompele, Jo

[51]

\section{Quantification and normalization of gene expression using SYBR Green I real-time RT-PCR}

Jo Vandesompele, Johanna Iso-Oja, Anne De Paepe \& Frank Speleman

Center for Medical Genetics 1K5, Ghent University Hospital, De Pintelaan 185, 9000 Ghent, Belgium

We have developed a two-step, real-time, quantitative assay, using the polymerase chain reaction with reverse transcription (RT-PCR) and based on SYBR Green I monitoring of PCR product accumulation, for quantification and normalization of gene expression levels. Because housekeeping gene expression can vary considerably among cell types or experimental conditions, our procedure uses multiple internal control genes for more accurate normalization of expression data, compared with traditional use of only one housekeeping gene. Owing to extensive accumulation of primer-dimers when no template control is used during one-step RT-PCR, we introduced a two-step protocol to eliminate this problem. This study further illustrates the prerequisite of DNase treatment of RNA samples before complementary DNA synthesis. The treatment also results in a significantly facilitated primer design for RT-PCR, as the positions of the primers are no longer important to control for genomic contamination. Real-time quantitative RT-PCR of DNase-treated samples and normalization using multiple internal controls is the method of choice for sensitive, accurate and large-scale measurements of gene expression levels.

Varley, Jenny

\section{Genotype and phenotype in carriers of germline TP53 mutations}

Jenny Varley ${ }^{1}$, Gareth Evans ${ }^{2}$, Jillian Birch ${ }^{3} \&$ Anna Kelsey ${ }^{4}$

${ }^{1}$ CRC Cancer Genetics Group, Paterson Institute for Cancer Research, Manchester, UK

${ }^{2}$ Department of Medical Genetics, St. Mary's Hospital, Manchester, UK ${ }^{3}$ CRC PFCRG and ${ }^{4}$ Department of Histopathology, Royal Manchester Children's Hospital, Manchester, UK

We collected data on a large cohort of families with features of Li-Fraumeni syndrome (LFS). To date we have identified germline TP53 mutations in 28 families: 20 of 25 classic LFS and 8 of 20 Li-Fraumeni-like. In addition we have identified germline mutations in 12 other individuals or families with tumors suggestive of LFS. Most mutations are missense or nonsense, but $7(18 \%)$ are splicing mutations. A survey of the literature showed that this is a considerably higher propor- 